A N N A LES

UNIVERSITATIS MARIAE CURIE-SKŁODOWSKA

LUBLIN - POLONIA

VOL. LXII, 2008

SECTIO A

$15-29$

M. K. AOUF, A. O. MOSTAFA and R. EL-ASHWAH

\title{
Differential sandwich theorems for analytic functions defined by some linear operators
}

\begin{abstract}
In this investigation, we obtain some applications of first order differential subordination and superordination results involving DziokSrivastava operator and other linear operators for certain normalized analytic functions. Some of our results improve previous results.
\end{abstract}

1. Introduction. Let $H(U)$ be the class of analytic functions in the unit disk $U=\{z \in C:|z|<1\}$ and let $H[a, k]$ be the subclass of $H(U)$ consisting of functions of the form:

$$
f(z)=a+a_{k} z^{k}+a_{k+1} z^{k+1} \ldots(a \in C) .
$$

For simplicity, let $H[a]=H[a, 1]$. Also, let $A$ be the subclass of $H(U)$ consisting of functions of the form:

$$
f(z)=z+a_{2} z^{2}+\ldots .
$$

If $f, g \in H(U)$, we say that $f$ is subordinate to $g$, written $f(z) \prec g(z)$ if there exists a Schwarz function $w(z)$, which (by definition) is analytic in $U$ with $w(0)=0$ and $|w(z)|<1$ for all $z \in U$, such that $f(z)=g(w(z))$, $z \in U$. Furthermore, if the function $g(z)$ is univalent in $U$, then we have the

2000 Mathematics Subject Classification. 30C45.

Key words and phrases. Analytic functions, differential subordination, superordination, sandwich theorems, Dziok-Srivastava operator. 
following equivalence, (cf., e.g., [4], [12]; see also [13]):

$$
f(z) \prec g(z)(z \in U) \Leftrightarrow f(0)=g(0) \text { and } f(U) \subset g(U) .
$$

Let $p, h \in H(U)$ and let $\varphi(r, s, t ; z): C^{3} \times U \rightarrow C$. If $p$ and $\varphi\left(p(z), z p^{\prime}(z)\right.$, $\left.z^{2} p^{\prime \prime}(z) ; z\right)$ are univalent and if $p$ satisfies the second order superordination

$$
h(z) \prec \varphi\left(p(z), z p^{\prime}(z), z^{2} p^{\prime \prime}(z) ; z\right),
$$

then $p$ is a solution of the differential superordination (1.3). Note that if $f$ is subordinate to $g$, then $g$ is superordinate to $f$. An analytic function $q$ is called a subordinant if $q(z) \prec p(z)$ for all $p$ satisfying (1.3). A univalent subordinant $\widetilde{q}$ that satisfies $q \prec \widetilde{q}$ for all subordinants of (1.3) is called the best subordinant. Recently Miller and Mocanu [14] obtained conditions on the functions $h, q$ and $\varphi$ for which the following implication holds:

$$
h(z) \prec \varphi\left(p(z), z p^{\prime}(z), z^{2} p^{\prime \prime}(z) ; z\right) \Rightarrow q(z) \prec p(z) .
$$

Using the results of Miller and Mocanu [14], Bulboača [3] considered certain classes of first order differential superordinations as well as superordination-preserving integral operators [5]. Ali et al. [1], have used the results of Bulboača [3] to obtain sufficient conditions for normalized analytic functions to satisfy:

$$
q_{1}(z) \prec \frac{z f^{\prime}(z)}{f(z)} \prec q_{2}(z),
$$

where $q_{1}$ and $q_{2}$ are given univalent functions in $U$. Also, Tuneski [18] obtained a sufficient condition for starlikeness of $f$ in terms of the quantity $\frac{f^{\prime \prime}(z) f(z)}{\left(f^{\prime}(z)\right)^{2}}$. Recently, Shanmugam et al. [16] obtained sufficient conditions for the normalized analytic function $f$ to satisfy

$$
q_{1}(z) \prec \frac{f(z)}{z f^{\prime}(z)} \prec q_{2}(z)
$$

and

$$
q_{1}(z) \prec \frac{z^{2} f^{\prime}(z)}{\{f(z)\}^{2}} \prec q_{2}(z) .
$$

They [16] also obtained results for functions defined by using CarlsonShaffer operator.

For complex numbers $\alpha_{1}, \alpha_{2}, \ldots, \alpha_{l}$ and $\beta_{1}, \beta_{2}, \ldots, \beta_{s}\left(\beta_{j} \notin Z_{0}^{-}=\right.$ $\{0,-1,-2, \ldots\} ; j=1,2, \ldots, s)$, we define the generalized hypergeometric function ${ }_{l} F_{s}\left(\alpha_{1}, \ldots, \alpha_{l} ; \beta_{1}, \ldots, \beta_{s} ; z\right)$ by (see, for example, [17]) by the following infinite series:

$$
\begin{aligned}
{ }_{l} F_{s}\left(\alpha_{1}, \ldots, \alpha_{l} ; \beta_{1}, \ldots, \beta_{s} ; z\right) & =\sum_{k=0}^{\infty} \frac{\left(\alpha_{1}\right)_{k} \ldots\left(\alpha_{l}\right)_{k}}{\left(\beta_{1}\right)_{k} \ldots\left(\beta_{s}\right)_{k}(1)_{k}} z^{k} \\
\left(l \leq s+1 ; s, l \in N_{0}\right. & =N \cup\{0\} ; z \in U),
\end{aligned}
$$


where

$$
(d)_{k}= \begin{cases}1 & (k=0 ; d \in C \backslash\{0\}) \\ d(d+1) \ldots(d+k-1) & (k \in N ; d \in C) .\end{cases}
$$

Dziok and Srivastava [9] (see also [10]) considered a linear operator $H_{l, s}\left(\alpha_{1}, \ldots, \alpha_{q} ; \beta_{1}, \ldots, \beta_{s}\right): A \rightarrow A$, defined by the following Hadamard product:

$$
H_{l, s}\left(\alpha_{1}, \ldots, \alpha_{l} ; \beta_{1}, \ldots, \beta_{s}\right) f(z)=\left[z_{l} F_{s}\left(\alpha_{1}, \ldots, \alpha_{l} ; \beta_{1}, \ldots, \beta_{s} ; z\right)\right] * f(z),
$$

$$
\left(l \leq s+1 ; s, l \in N_{0} ; z \in U\right) .
$$

We observe that for a function $f$ of the form (1.2), we have

$$
\begin{aligned}
& H_{l, s}\left(\alpha_{1}, \ldots, \alpha_{l} ; \beta_{1}, \ldots, \beta_{s}\right) f(z) \\
& \quad=z+\sum_{k=2}^{\infty} \frac{\left(\alpha_{1}\right)_{k-1} \ldots\left(\alpha_{l}\right)_{k-1}}{\left(\beta_{1}\right)_{k-1} \ldots\left(\beta_{s}\right)_{k-1}(1)_{k-1}} a_{k} z^{k} .
\end{aligned}
$$

If, for convenience, we write

$$
H_{l, s}\left(\alpha_{1}\right)=H_{l, s}\left(\alpha_{1}, \ldots, \alpha_{l} ; \beta_{1}, \ldots, \beta_{s}\right),
$$

then one can easily verify from the definition (1.7) that

$$
z\left(H_{l, s}\left(\alpha_{1}\right) f(z)\right)^{\prime}=\alpha_{1} H_{l, s}\left(\alpha_{1}+1\right) f(z)-\left(\alpha_{1}-1\right) H_{l, s}\left(\alpha_{1}\right) f(z)
$$

$(f(z) \in A)$.

It should be remarked that the linear operator $H_{l, s}\left(\alpha_{1}\right) f(z)$ is a generalization of many other linear operators considered earlier. In particular, for $f \in A$, we have:

(i) $H_{2,1}(a, 1 ; c) f(z)=L(a, c) f(z)(a>0 ; c>0)$, where $L(a, c)$ is the Carlson-Shaffer operator (see [6]);

(ii) $H_{2,1}(\lambda+1, c ; a) f(z)=I^{\lambda}(a, c) f(z)\left(a, c \in R \backslash Z_{0}^{-} ; \lambda>-1\right)$, where $I^{\lambda}(a, c) f(z)$ is the Cho-Kwon-Srivastava operator (see [7]);

(iii) $H_{2,1}(\mu, 1 ; \lambda+1) f(z)=I_{\lambda, \mu} f(z)(\lambda>-1 ; \mu>0)$, where $I_{\lambda, \mu} f(z)$ is the Choi-Saigo-Srivastava operator (see [8]);

(iv) $H_{2,1}(\mu+1,1 ; \mu+2) f(z)=F_{\mu}(f)(z)=\frac{\mu+1}{z^{\mu}} \int_{0}^{z} t^{\mu-1} f(t) d t(\mu>-1)$ where $F_{\mu}(f)(z)$ is the Libera operator (see [11] and [2]);

(v) $H_{2,1}(\delta+1,1 ; 1) f(z)=D^{\delta} f(z)(\delta>-1)$, where $D^{\delta} f(z)$ is the $\delta$ Ruscheweyh derivative of $f(z)$ (see [15]).

In this paper, we obtain sufficient conditions for the normalized analytic function $f$ defined by using Dziok-Srivastava operator to satisfy:

$$
q_{1}(z) \prec \frac{H_{l, s}\left(\alpha_{1}\right) f(z)}{H_{l, s}\left(\alpha_{1}+1\right) f(z)} \prec q_{2}(z)
$$

and

$$
q_{1}(z) \prec \frac{H_{l, s}\left(\alpha_{1}+1\right) f(z)}{\left\{H_{l, s}\left(\alpha_{1}\right) f(z)\right\}^{2}} \prec q_{2}(z)
$$


and $q_{1}$ and $q_{2}$ are given univalent functions in $U$.

2. Definitions and preliminaries. In order to prove our results, we shall make use of the following known results.

Definition 1 ([14]). Denote by $Q$, the set of all functions $f$ that are analytic and injective on $\bar{U} \backslash E(f)$, where

$$
E(f)=\left\{\xi \in \partial U: \lim _{z \rightarrow \xi} f(z)=\infty\right\},
$$

and are such that $f^{\prime}(\xi) \neq 0$ for $\xi \in \partial U \backslash E(f)$.

Lemma 1 ([14]). Let $q$ be univalent in the unit disk $U$ and $\theta$ and $\varphi$ be analytic in a domain $D$ containing $q(U)$ with $\varphi(w) \neq 0$ when $w \in q(U)$. Set

$$
\psi(z)=z q^{\prime}(z) \varphi(q(z)) \text { and } h(z)=\theta(q(z))+\psi(z) .
$$

Suppose that

(i) $\psi(z)$ is starlike univalent in $U$,

(ii) $\operatorname{Re}\left\{\frac{z h^{\prime}(z)}{\psi(z)}\right\}>0$ for $z \in U$.

If $p$ is analytic with $p(0)=q(0), p(U) \subseteq D$ and

$$
\theta(p(z))+z p^{\prime}(z) \varphi(p(z)) \prec \theta(q(z))+z q^{\prime}(z) \varphi(q(z)),
$$

then $p(z) \prec q(z)$ and $q$ is the best dominant.

Taking $\theta(w)=\alpha w$ and $\varphi(w)=\gamma$ in Lemma 1, Shanmugam et al. [16] obtained the following lemma.

Lemma 2 ([16]). Let $q$ be univalent in $U$ with $q(0)=1$. Let $\alpha \in C$; $\gamma \in C^{*}=C \backslash\{0\}$, further assume that

$$
\operatorname{Re}\left\{1+\frac{z q^{\prime \prime}(z)}{q^{\prime}(z)}\right\}>\max \{0,-\operatorname{Re}(\alpha / \gamma)\} .
$$

If $p$ is analytic in $U$, and

$$
\alpha p(z)+\gamma z p^{\prime}(z) \prec \alpha q(z)+\gamma z q^{\prime}(z),
$$

then $p \prec q$ and $q$ is the best dominant.

Lemma 3 ([3]). Let $q$ be convex univalent in $U$ and $\vartheta$ and $\phi$ be analytic in a domain $D$ containing $q(U)$. Suppose that

(i) $\operatorname{Re}\left\{\vartheta^{\prime}(q(z)) / \phi(q(z))\right\}>0$ for $z \in U$,

(ii) $\psi(z)=z q^{\prime}(z) \phi(q(z))$ is starlike univalent in $U$.

If $p(z) \in H[q(0), 1] \cap Q$, with $p(U) \subseteq D$, and $\vartheta(p(z))+z p^{\prime}(z) \phi(p(z))$ is univalent in $U$ and

$$
\vartheta(q(z))+z q^{\prime}(z) \phi(q(z)) \prec \vartheta(p(z))+z p^{\prime}(z) \phi(p(z)),
$$

then $q(z) \prec p(z)$ and $q$ is the best subordinant. 
Taking $\theta(w)=\alpha w$ and $\varphi(w)=\gamma$ in Lemma 3, Shanmugam et al. [16] obtained the following lemma.

Lemma 4 ([16]). Let $q$ be convex univalent in $U, q(0)=1$. Let $\alpha \in C$, $\gamma \in C^{*}$ and $\operatorname{Re}\{\alpha / \gamma\}>0$. If $p \in H[q(0), 1] \cap Q, \alpha p(z)+\gamma z p^{\prime}(z)$ is univalent in $U$ and

$$
\alpha q(z)+\gamma z q^{\prime}(z) \prec \alpha p(z)+\gamma z p^{\prime}(z),
$$

then $q \prec p$ and $q$ is the best subordinant.

\section{Applications to Dziok-Srivastava operator and sandwich theo-} rems.

Theorem 1. Let $q$ be convex univalent in $U$ with $q(0)=1, \gamma \in C^{*}$. Further, assume that

$$
\operatorname{Re}\left\{1+\frac{z q^{\prime \prime}(z)}{q^{\prime}(z)}\right\}>\max \{0,-\operatorname{Re}(1 / \gamma)\} .
$$

If $f \in A, H_{l, s}\left(\alpha_{1}+1\right) f(z) \neq 0$ for $0<|z|<1$, and

$$
\begin{array}{r}
\gamma \alpha_{1}+(1+\gamma) \frac{H_{l, s}\left(\alpha_{1}\right) f(z)}{H_{l, s}\left(\alpha_{1}+1\right) f(z)} \\
-\gamma\left(1+\alpha_{1}\right) \frac{H_{l, s}\left(\alpha_{1}+2\right) f(z) H_{l, s}\left(\alpha_{1}\right) f(z)}{\left\{H_{l, s}\left(\alpha_{1}+1\right) f(z)\right\}^{2}} \\
\prec q(z)+\gamma z q^{\prime}(z),
\end{array}
$$

then

$$
\frac{H_{l, s}\left(\alpha_{1}\right) f(z)}{H_{l, s}\left(\alpha_{1}+1\right) f(z)} \prec q(z)
$$

and $q$ is the best dominant.

Proof. Define a function $p$ by

$$
p(z)=\frac{H_{l, s}\left(\alpha_{1}\right) f(z)}{H_{l, s}\left(\alpha_{1}+1\right) f(z)} \quad(z \in U) .
$$

Then the function $p$ is analytic in $U$ and $p(0)=1$. Therefore, differentiating (3.3) logarithmically with respect to $z$ and using the identity (1.9) in the resulting equation, we have

$$
\begin{gathered}
\gamma \alpha_{1}+(1+\gamma) \frac{H_{l, s}\left(\alpha_{1}\right) f(z)}{H_{l, s}\left(\alpha_{1}+1\right) f(z)}-\gamma\left(1+\alpha_{1}\right) \frac{H_{l, s}\left(\alpha_{1}+2\right) f(z) H_{l, s}\left(\alpha_{1}\right) f(z)}{\left\{H_{l, s}\left(\alpha_{1}+1\right) f(z)\right\}^{2}} \\
=p(z)+\gamma z p^{\prime}(z),
\end{gathered}
$$

that is,

$$
p(z)+\gamma z p^{\prime}(z) \prec q(z)+\gamma z q^{\prime}(z)
$$

and therefore, the theorem follows by applying Lemma 2 .

Putting $q(z)=(1+A z) /(1+B z)(-1 \leq B<A \leq 1)$ in Theorem 1 , we have the following corollary. 
Corollary 1. If $f(z) \in A$ and $\gamma \in C^{*}$ satisfy

$$
\begin{gathered}
\gamma \alpha_{1}+(1+\gamma) \frac{H_{l, s}\left(\alpha_{1}\right) f(z)}{H_{l, s}\left(\alpha_{1}+1\right) f(z)}-\gamma\left(1+\alpha_{1}\right) \frac{H_{l, s}\left(\alpha_{1}+2\right) f(z) H_{l, s}\left(\alpha_{1}\right) f(z)}{\left\{H_{l, s}\left(\alpha_{1}+1\right) f(z)\right\}^{2}} \\
\prec \gamma \frac{(A-B) z}{(1+B z)^{2}}+\frac{1+A z}{1+B z},
\end{gathered}
$$

then

$$
\frac{H_{l, s}\left(\alpha_{1}\right) f(z)}{H_{l, s}\left(\alpha_{1}+1\right) f(z)} \prec \frac{1+A z}{1+B z} .
$$

Putting $A=1, B=-1$ and $q(z)=\frac{1+z}{1-z}$ in Corollary 1, we have

Corollary 2. If $f(z) \in A$ and $\gamma \in C^{*}$ satisfy

$$
\begin{gathered}
\gamma \alpha_{1}+(1+\gamma) \frac{H_{l, s}\left(\alpha_{1}\right) f(z)}{H_{l, s}\left(\alpha_{1}+1\right) f(z)}-\gamma\left(1+\alpha_{1}\right) \frac{H_{l, s}\left(\alpha_{1}+2\right) f(z) H_{l, s}\left(\alpha_{1}\right) f(z)}{\left\{H_{l, s}\left(\alpha_{1}+1\right) f(z)\right\}^{2}} \\
\prec \frac{2 \gamma z}{(1-z)^{2}}+\frac{1+z}{1-z},
\end{gathered}
$$

then

$$
\operatorname{Re}\left\{\frac{H_{l, s}\left(\alpha_{1}\right) f(z)}{H_{l, s}\left(\alpha_{1}+1\right) f(z)}\right\}>0 .
$$

Taking $\alpha_{1}=a>0, \beta_{1}=c>0, \alpha_{j}=1(j=2, \ldots, s+1)$ and $\beta_{j}=1$ $(j=2, \ldots, s)$, in Theorem 1 , we have the following corollary which improves the result of Shanmugam et al. [16, Theorem 4.1].

Corollary 3. Let $q$ be convex univalent in $U$ with $q(0)=1, \gamma \in C^{*}$. Further, assume that (3.1) holds. If $f \in A$, and

$$
\begin{gathered}
\gamma a+(1+\gamma) \frac{L(a, c) f(z)}{L(a+1, c)} f(z)-\gamma(1+a) \frac{L(a+2, c) f(z) L(a, c) f(z)}{\{L(a+1, c) f(z)\}^{2}} \\
\prec q(z)+\gamma z q^{\prime}(z),
\end{gathered}
$$

then

$$
\frac{L(a, c) f(z)}{L(a+1, c) f(z)} \prec q(z)
$$

and $q$ is the best dominant.

Taking $\alpha_{1}=\lambda+1, \alpha_{2}=c, \beta_{1}=a\left(a, c \in R \backslash Z_{o}^{-} ; \lambda>-1\right), \alpha_{j}=1$ $(j=3, \ldots, s+1)$ and $\beta_{j}=1(j=2, \ldots, s)$, in Theorem 1 , we have

Corollary 4. Let $q$ be convex univalent in $U$ with $q(0)=1, \gamma \in C^{*}$. Further, assume that (3.1) holds. If $f \in A$, and

$$
\begin{gathered}
\gamma(\lambda+1)+(1+\gamma) \frac{I^{\lambda}(a, c) f(z)}{I^{\lambda+1}(a, c) f(z)}-\gamma(\lambda+2) \frac{I^{\lambda+2}(a, c) f(z) I^{\lambda}(a, c) f(z)}{\left\{I^{\lambda+1}(a, c) f(z)\right\}^{2}} \\
\prec q(z)+\gamma z q^{\prime}(z),
\end{gathered}
$$


then

$$
\frac{I^{\lambda}(a, c) f(z)}{I^{\lambda+1}(a, c) f(z)} \prec q(z)
$$

and $q$ is the best dominant.

Taking $\alpha_{1}=\mu, \beta_{1}=\lambda+1(\lambda>-1 ; \mu>0), \alpha_{j}=1(j=2, \ldots, s+1)$ and $\beta_{j}=1(j=2, \ldots, s)$ in Theorem 1 , we have

Corollary 5. Let $q$ be convex univalent in $U$ with $q(0)=1, \gamma \in C^{*}$. Further, assume that (3.1) holds. If $f \in A$, and

$$
\gamma \mu+(1+\gamma) \frac{I_{\lambda, \mu} f(z)}{I_{\lambda, \mu+1} f(z)}-\gamma(\mu+1) \frac{I_{\lambda, \mu+2} f(z) I_{\lambda, \mu} f(z)}{\left\{I_{\lambda, \mu} f(z)\right\}^{2}} \prec q(z)+\gamma z q^{\prime}(z),
$$

then

$$
\frac{I_{\lambda, \mu} f(z)}{I_{\lambda, \mu+1} f(z)} \prec q(z)
$$

and $q$ is the best dominant.

Taking $\alpha_{1}=\mu+1, \beta_{1}=\mu+2(\mu>-1), \alpha_{j}=1(j=2, \ldots, s+1)$ and $\beta_{j}=1(j=2, \ldots, s)$ in Theorem 1 , we have

Corollary 6. Let $q$ be convex univalent in $U$ with $q(0)=1, \gamma \in C^{*}$. Further, assume that (3.1) holds. If $f \in A$, and

$$
\gamma(1+\mu)+(1-\gamma \mu) \frac{F_{\mu} f(z)}{f(z)}-\gamma \frac{z f^{\prime}(z) F_{\mu} f(z)}{\{f(z)\}^{2}} \prec q(z)+\gamma z q^{\prime}(z),
$$

then

$$
\frac{F_{\mu} f(z)}{f(z)} \prec q(z)
$$

and $q$ is the best dominant.

Now, by appealing to Lemma 4 it can be easily prove the following theorem.

Theorem 2. Let $q$ be convex univalent in $U$. Let $\gamma \in C$ with $\operatorname{Re} \gamma>0$. If $f \in A, \frac{H_{l, s}\left(\alpha_{1}\right) f(z)}{H_{l, s}\left(\alpha_{1}+1\right) f(z)} \in H[1,1] \cap Q$,

$$
\gamma \alpha_{1}+(1+\gamma) \frac{H_{l, s}\left(\alpha_{1}\right) f(z)}{H_{l, s}\left(\alpha_{1}+1\right) f(z)}-\gamma\left(1+\alpha_{1}\right) \frac{H_{l, s}\left(\alpha_{1}+2\right) f(z) H_{l, s}\left(\alpha_{1}\right) f(z)}{\left\{H_{l, s}\left(\alpha_{1}+1\right) f(z)\right\}^{2}}
$$

is univalent in $U$, and

$$
\begin{aligned}
q(z)+\gamma z q^{\prime}(z) \prec & \gamma \alpha_{1}+(1+\gamma) \frac{H_{l, s}\left(\alpha_{1}\right) f(z)}{H_{l, s}\left(\alpha_{1}+1\right) f(z)} \\
& -\gamma\left(1+\alpha_{1}\right) \frac{H_{l, s}\left(\alpha_{1}+2\right) f(z) H_{l, s}\left(\alpha_{1}\right) f(z)}{\left\{H_{l, s}\left(\alpha_{1}+1\right) f(z)\right\}^{2}},
\end{aligned}
$$


then

$$
q(z) \prec \frac{H_{l, s}\left(\alpha_{1}\right) f(z)}{H_{l, s}\left(\alpha_{1}+1\right) f(z)}
$$

and $q$ is the best subordinant.

Taking $\alpha_{1}=a>0, \beta_{1}=c>0, \alpha_{j}=1(j=2, \ldots, s+1)$ and $\beta_{j}=1$ $(j=2, \ldots, s)$ in Theorem 2 , we have the following corollary which improve the result of Shanmugam et al. [16, Theorem 4.2].

Corollary 7. Let $q$ be convex univalent in $U$. Let $\gamma \in C$ with $\operatorname{Re} \gamma>0$. If $f \in A, \frac{L(a, c) f(z)}{L(a+1, c) f(z)} \in H[1,1] \cap Q$,

$$
\gamma a+(1+\gamma) \frac{L(a, c) f(z)}{L(a+1, c) f(z)}-\gamma(1+a) \frac{L(a+2, c) f(z) L(a, c) f(z)}{\{L(a+1, c) f(z)\}^{2}}
$$

is univalent in $U$, and

$$
\begin{aligned}
q(z)+\gamma z q^{\prime}(z) \prec & \gamma a+(1+\gamma) \frac{L(a, c) f(z)}{L(a+1, c) f(z)} \\
& -\gamma(1+a) \frac{L(a+2, c) f(z) L(a, c) f(z)}{\{L(a+1, c) f(z)\}^{2}}
\end{aligned}
$$

then

$$
q(z) \prec \frac{L(a, c) f(z)}{L(a+1, c) f(z)}
$$

and $q$ is the best subordinant.

Taking $\alpha_{1}=\lambda+1, \alpha_{2}=c, \beta_{1}=a\left(a, c \in R \backslash Z_{o}^{-} ; \lambda>-1\right), \alpha_{j}=1$ $(j=3, \ldots, s+1)$ and $\beta_{j}=1(j=2, \ldots, s)$, in Theorem 2 , we have

Corollary 8. Let $q$ be convex univalent in $U$. Let $\gamma \in C$ with $\operatorname{Re} \gamma>0$. If $f \in A, \frac{I^{\lambda}(a, c) f(z)}{I^{\lambda+1}(a, c) f(z)} \in H[1,1] \cap Q$,

$$
\gamma(\lambda+1)+(1+\gamma) \frac{I^{\lambda}(a, c) f(z)}{I^{\lambda+1}(a, c) f(z)}-\gamma(\lambda+2) \frac{I^{\lambda+2}(a, c) f(z) I^{\lambda}(a, c) f(z)}{\left\{I^{\lambda+1}(a, c) f(z)\right\}^{2}}
$$

is univalent in $U$, and

$$
\begin{aligned}
q(z)+\gamma z q^{\prime}(z) \prec & \gamma(\lambda+1)+(1+\gamma) \frac{I^{\lambda}(a, c) f(z)}{I^{\lambda+1}(a, c) f(z)} \\
& -\gamma(\lambda+2) \frac{I^{\lambda+2}(a, c) f(z) I^{\lambda}(a, c) f(z)}{\left\{I^{\lambda+1}(a, c) f(z)\right\}^{2}}
\end{aligned}
$$

then

$$
q(z) \prec \frac{I^{\lambda}(a, c) f(z)}{I^{\lambda+1}(a, c) f(z)}
$$

and $q$ is the best subordinant. 
Taking $\alpha_{1}=\mu, \beta_{1}=\lambda+1(\lambda>-1 ; \mu>0), \alpha_{j}=1(j=2, \ldots, s+1)$ and $\beta_{j}=1(j=2, \ldots, s)$, in Theorem 2 , we have

Corollary 9. Let $q$ be convex univalent in $U$. Let $\gamma \in C$ with $\operatorname{Re} \gamma>0$. If $f \in A, \frac{I_{\lambda, \mu} f(z)}{I_{\lambda, \mu+1} f(z)} \in H[1,1] \cap Q$,

$$
\gamma \mu+(1+\gamma) \frac{I_{\lambda, \mu} f(z)}{I_{\lambda, \mu+1} f(z)}-\gamma(\mu+1) \frac{I_{\lambda, \mu+2} f(z) I_{\lambda, \mu} f(z)}{\left\{I_{\lambda, \mu} f(z)\right\}^{2}}
$$

is univalent in $U$, and

$$
q(z)+\gamma z q^{\prime}(z) \prec \gamma \mu+(1+\gamma) \frac{I_{\lambda, \mu} f(z)}{I_{\lambda, \mu+1} f(z)}-\gamma(\mu+1) \frac{I_{\lambda, \mu+2} f(z) I_{\lambda, \mu} f(z)}{\left\{I_{\lambda, \mu} f(z)\right\}^{2}},
$$

then

$$
q(z) \prec \frac{I_{\lambda, \mu} f(z)}{I_{\lambda, \mu+1} f(z)}
$$

and $q$ is the best subordinant.

Taking $\alpha_{1}=\mu+1, \beta_{1}=\mu+2(\mu>-1), \alpha_{j}=1(j=2, \ldots, s+1)$ and $\beta_{j}=1(j=2, \ldots, s)$, in Theorem 2 , we have

Corollary 10. Let $q$ be convex univalent in $U$. Let $\gamma \in C$ with $\operatorname{Re} \gamma>0$. If $f \in A, \frac{F_{\mu} f(z)}{f(z)} \in H[1,1] \cap Q$,

$$
\gamma(1+\mu)+(1-\gamma \mu) \frac{F_{\mu} f(z)}{f(z)}-\gamma \frac{z f^{\prime}(z) F_{\mu} f(z)}{\{f(z)\}^{2}}
$$

is univalent in $U$, and

$$
q(z)+\gamma z q^{\prime}(z) \prec \gamma(1+\mu)+(1-\gamma \mu) \frac{F_{\mu} f(z)}{f(z)}-\gamma \frac{z f^{\prime}(z) F_{\mu} f(z)}{\{f(z)\}^{2}},
$$

then

$$
q(z) \prec \frac{F_{\mu} f(z)}{f(z)}
$$

and $q$ is the best dominant.

Combining Theorem 1 and Theorem 2, we get the following sandwich theorem.

Theorem 3. Let $q_{1}$ be convex univalent in $U, \gamma \in C$ with $\operatorname{Re} \gamma>0, q_{2}$ be univalent in $U, q_{2}(0)=1$ and satisfies (3.1). If $f \in A, \frac{H_{l, s}\left(\alpha_{1}\right) f(z)}{H_{l, s}\left(\alpha_{1}+1\right) f(z)} \in$ $H[1,1] \cap Q$,

$$
\gamma \alpha_{1}+(1+\gamma) \frac{H_{l, s}\left(\alpha_{1}\right) f(z)}{H_{l, s}\left(\alpha_{1}+1\right) f(z)}-\gamma\left(1+\alpha_{1}\right) \frac{H_{l, s}\left(\alpha_{1}+2\right) f(z) H_{l, s}\left(\alpha_{1}\right) f(z)}{\left\{H_{l, s}\left(\alpha_{1}+1\right) f(z)\right\}^{2}}
$$


is univalent in $U$, and

$$
\begin{aligned}
& q_{1}(z)+\gamma z q_{1}^{\prime}(z) \prec \gamma \alpha_{1}+(1+\gamma) \frac{H_{l, s}\left(\alpha_{1}\right) f(z)}{H_{l, s}\left(\alpha_{1}+1\right) f(z)} \\
&-\gamma\left(1+\alpha_{1}\right) \frac{H_{l, s}\left(\alpha_{1}+2\right) f(z) H_{l, s}\left(\alpha_{1}\right) f(z)}{\left\{H_{l, s}\left(\alpha_{1}+1\right) f(z)\right\}^{2}} \\
& \prec q_{2}(z)+\gamma z q_{2}^{\prime}(z),
\end{aligned}
$$

then

$$
q_{1}(z) \prec \frac{H_{l, s}\left(\alpha_{1}\right) f(z)}{H_{l, s}\left(\alpha_{1}+1\right) f(z)} \prec q_{2}(z)
$$

and $q_{1}$ and $q_{2}$ are, respectively, the best subordinant and the best dominant.

4. Remarks. Combining: (i) Corollary 3 and Corollary 7; (ii) Corollary 4 and Corollary 8; (iii) Corollary 5 and Corollary 9; (iv) Corollary 6 and Corollary 10, we obtain similar sandwich theorems for the corresponding operators.

Theorem 4. Let $q$ be convex univalent in $U, \gamma \in C^{*}$. Further, assume that (3.1) holds. If $f \in A$ satisfies

$$
\begin{gathered}
{\left[1+\gamma\left(\alpha_{1}-1\right)\right] \frac{z H_{l, s}\left(\alpha_{1}+1\right) f(z)}{\left[H_{l, s}\left(\alpha_{1}\right) f(z)\right]^{2}}+\gamma\left(1+\alpha_{1}\right) \frac{z H_{l, s}\left(\alpha_{1}+2\right) f(z)}{\left[H_{l, s}\left(\alpha_{1}\right) f(z)\right]^{2}}} \\
-2 \gamma \alpha_{1} \frac{z\left[H_{l, s}\left(\alpha_{1}+1\right) f(z)\right]^{2}}{\left[H_{l, s}\left(\alpha_{1}\right) f(z)\right]^{3}} \prec q(z)+\gamma z q^{\prime}(z),
\end{gathered}
$$

then

$$
\frac{z H_{l, s}\left(\alpha_{1}+1\right) f(z)}{\left[H_{l, s}\left(\alpha_{1}\right) f(z)\right]^{2}} \prec q(z)
$$

and $q$ is the best dominant.

Proof. Define the function $p(z)$ by

$$
p(z)=\frac{z H_{l, s}\left(\alpha_{1}+1\right) f(z)}{\left[H_{l, s}\left(\alpha_{1}\right) f(z)\right]^{2}} \quad(z \in U) .
$$

Then, simple computations show that

$$
\begin{aligned}
p(z)+\gamma z p^{\prime}(z)= & {\left[1+\gamma\left(\alpha_{1}-1\right)\right] \frac{z H_{l, s}\left(\alpha_{1}+1\right) f(z)}{\left[H_{l, s}\left(\alpha_{1}\right) f(z)\right]^{2}} } \\
& +\gamma\left(1+\alpha_{1}\right) \frac{z H_{l, s}\left(\alpha_{1}+2\right) f(z)}{\left[H_{l, s}\left(\alpha_{1}\right) f(z)\right]^{2}}-2 \gamma \alpha_{1} \frac{z\left[H_{l, s}\left(\alpha_{1}+1\right) f(z)\right]^{2}}{\left[H_{l, s}\left(\alpha_{1}\right) f(z)\right]^{3}} .
\end{aligned}
$$

Applying Lemma 2, the theorem follows.

Taking $\alpha_{1}=a>0, \beta_{1}=c>0, \alpha_{j}=1(j=2, \ldots, s+1)$ and $\beta_{j}=1$ $(j=2, \ldots, s)$ in Theorem 4 , we have the following corollary which improves the result of Shanmugam et al. [16, Theorem 4.4]. 
Corollary 11. Let $q$ be convex univalent in $U, \gamma \in C^{*}$. Further, assume that (3.1) holds. If $f \in A$ satisfies

$$
\begin{aligned}
& {[1+\gamma(a-1)] } \frac{z L(a+1, c) f(z)}{[L(a, c) f(z)]^{2}} \\
&+\gamma(1+a) \frac{z L(a+2, c) f(z)}{[L(a, c) f(z)]^{2}}-2 \gamma a \frac{z[L(a+1, c) f(z)]^{2}}{[L(a, c) f(z)]^{3}} \\
& \prec q(z)+\gamma z q^{\prime}(z),
\end{aligned}
$$

then

$$
\frac{z L(a+1, c) f(z)}{[L(a, c) f(z)]^{2}} \prec q(z)
$$

and $q$ is the best dominant.

Taking $\alpha_{1}=\lambda+1, \alpha_{2}=c, \beta_{1}=a\left(a, c \in R \backslash Z^{-} ; \lambda>-1\right), \alpha_{j}=1$ $(j=3, \ldots, s+1)$ and $\beta_{j}=1(j=2, \ldots, s)$, in Theorem 4 , we have

Corollary 12. Let $q$ be convex univalent in $U, \gamma \in C^{*}$. Further, assume that (3.1) holds. If $f \in A$ satisfies

$$
\begin{aligned}
& {[1+\gamma(\lambda-1)] \frac{z I^{\lambda+1}(a, c) f(z)}{\left[I^{\lambda}(a, c) f(z)\right]^{2}} } \\
&+\gamma(\lambda+2) \frac{z I^{\lambda+2}(a, c) f(z)}{\left[I^{\lambda}(a, c) f(z)\right]^{2}}-2 \gamma(\lambda+1) \frac{z\left[I^{\lambda+1}(a, c) f(z)\right]^{2}}{\left\{I^{\lambda}(a, c) f(z)\right\}^{3}} \\
& \prec q(z)+\gamma z q^{\prime}(z),
\end{aligned}
$$

then

$$
\frac{z I^{\lambda+1}(a, c) f(z)}{\left[I^{\lambda}(a, c) f(z)\right]^{2}} \prec q(z)
$$

and $q$ is the best dominant.

Taking $\alpha_{1}=\mu, \beta_{1}=\lambda+1(\lambda>-1 ; \mu>0), \alpha_{j}=1(j=2, \ldots, s+1)$ and $\beta_{j}=1(j=2, \ldots, s)$, in Theorem 4 , we have

Corollary 13. Let $q$ be convex univalent in $U, \gamma \in C^{*}$. Further, assume that (3.1) holds. If $f \in A$ satisfies

$$
\begin{aligned}
& {[1+\gamma(\mu-1)] \frac{z I_{\lambda, \mu+1} f(z)}{\left[I_{\lambda, \mu} f(z)\right]^{2}} } \\
&+\gamma(\mu+1) \frac{z I_{\lambda, \mu+2} f(z)}{\left[I_{\lambda, \mu} f(z)\right]^{2}}-2 \gamma \mu \frac{z\left[I_{\lambda, \mu+1} f(z)\right]^{2}}{\left\{I_{\lambda, \mu} f(z)\right\}^{3}} \\
& \prec q(z)+\gamma z q^{\prime}(z),
\end{aligned}
$$

then

and $q$ is the best dominant.

$$
\frac{z I_{\lambda, \mu+1} f(z)}{\left[I_{\lambda, \mu} f(z)\right]^{2}} \prec q(z)
$$


Taking $\alpha_{1}=\mu+1, \beta_{1}=\mu+2(\mu>-1), \alpha_{j}=1(j=2, \ldots, s+1)$ and $\beta_{j}=1(j=2, \ldots, s)$, in Theorem 4 , we have

Corollary 14. Let $q$ be convex univalent in $U, \gamma \in C^{*}$. Further, assume that (3.1) holds. If $f \in A$ satisfies

$[1+\gamma(1+2 \mu)] \frac{z f(z)}{\left[F_{\mu} f(z)\right]^{2}}+\gamma \frac{z^{2} f^{\prime}(z)}{\left[F_{\mu} f(z)\right]^{2}}-2 \gamma(\mu+1) \frac{z(f(z))^{2}}{\left[F_{\mu} f(z)\right]^{3}} \prec q(z)+\gamma z q^{\prime}(z)$,

then

$$
\frac{z f(z)}{\left[F_{\mu} f(z)\right]^{2}} \prec q(z)
$$

and $q$ is the best dominant.

Theorem 5. Let $q$ be convex univalent in $U$. Let $\gamma \in C$ with $\operatorname{Re} \gamma>0$. If $f \in A, \frac{z H_{l, s}\left(\alpha_{1}+1\right) f(z)}{\left[H_{l, s}\left(\alpha_{1}\right) f(z)\right]^{2}} \in H[1,1] \cap Q$,

$$
\begin{gathered}
{\left[1+\gamma\left(\alpha_{1}-1\right)\right] \frac{z H_{l, s}\left(\alpha_{1}+1\right) f(z)}{\left[H_{l, s}\left(\alpha_{1}\right) f(z)\right]^{2}}+\gamma\left(1+\alpha_{1}\right) \frac{z H_{l, s}\left(\alpha_{1}+2\right) f(z)}{\left[H_{l, s}\left(\alpha_{1}\right) f(z)\right]^{2}}} \\
-2 \gamma \alpha_{1} \frac{z\left[H_{l, s}\left(\alpha_{1}+1\right) f(z)\right]^{2}}{\left[H_{l, s}\left(\alpha_{1}\right) f(z)\right]^{3}}
\end{gathered}
$$

is univalent in $U$, and

$$
\begin{gathered}
q(z)+\gamma z q^{\prime}(z) \prec\left[1+\gamma\left(\alpha_{1}-1\right)\right] \frac{z H_{l, s}\left(\alpha_{1}+1\right) f(z)}{\left[H_{l, s}\left(\alpha_{1}\right) f(z)\right]^{2}}+\gamma\left(1+\alpha_{1}\right) \frac{z H_{l, s}\left(\alpha_{1}+2\right) f(z)}{\left[H_{l, s}\left(\alpha_{1}\right) f(z)\right]^{2}} \\
-2 \gamma \alpha_{1} \frac{z\left[H_{l, s}\left(\alpha_{1}+1\right) f(z)\right]^{2}}{\left[H_{l, s}\left(\alpha_{1}\right) f(z)\right]^{3}}
\end{gathered}
$$

then

$$
q(z) \prec \frac{z H_{l, s}\left(\alpha_{1}+1\right) f(z)}{\left[H_{l, s}\left(\alpha_{1}\right) f(z)\right]^{2}}
$$

and $q$ is the best subordinant.

Proof. The proof follows by applying Lemma 4.

Taking $\alpha_{1}=a>0, \beta_{1}=c>0, \alpha_{j}=1(j=2, \ldots, s+1)$ and $\beta_{j}=1$ $(j=2, \ldots, s)$ in Theorem 5 , we have the following corollary which improve the result of Shanmugam et al. [16, Theorem 4.5].

Corollary 15. Let $q$ be convex univalent in $U$. Let $\gamma \in C$ with $\operatorname{Re} \gamma>0$. If $f \in A, \frac{z L(a+1, c) f(z)}{[L(a, c) f(z)]^{2}} \in H[1,1] \cap Q$,

$$
\begin{aligned}
{[1+\gamma(a-1)] } & \frac{z L(a+1, c) f(z)}{[L(a, c) f(z)]^{2}} \\
& +\gamma(1+a) \frac{z L(a+2, c) f(z)}{[L(a, c) f(z)]^{2}}-2 \gamma a \frac{z[L(a+1, c) f(z)]^{2}}{[L(a, c) f(z)]^{3}}
\end{aligned}
$$


is univalent in $U$, and

$$
\begin{gathered}
q(z)+\gamma z q^{\prime}(z) \prec\left[1+\gamma\left(\alpha_{1}-1\right)\right] \frac{z L(a+1, c) f(z)}{[L(a, c) f(z)]^{2}}+\gamma(1+a) \frac{z L(a+2, c) f(z)}{[L(a, c) f(z)]^{2}} \\
-2 \gamma a \frac{z[L(a+1, c) f(z)]^{2}}{[L(a, c) f(z)]^{3}},
\end{gathered}
$$

then

and $q$ is the best subordinant.

$$
q(z) \prec \frac{z L(a+1, c) f(z)}{[L(a, c) f(z)]^{2}}
$$

Combining Theorem 4 and Theorem 5, we get the following sandwich theorem.

Theorem 6. Let $q_{1}$ be convex univalent in $U, \gamma \in C$ with $\operatorname{Re}\{\gamma\}>0, q_{2}$ be univalent in $U, q_{2}(0)=1$ and satisfies (3.1). If $f \in A, \frac{z H_{l, s}\left(\alpha_{1}+1\right) f(z)}{\left[H_{l, s}\left(\alpha_{1}\right) f(z)\right]^{2}} \in$ $H[1,1] \cap Q$,

$$
\begin{aligned}
{\left[1+\gamma\left(\alpha_{1}-1\right)\right] } & \frac{z H_{l, s}\left(\alpha_{1}+1\right) f(z)}{\left[H_{l, s}\left(\alpha_{1}\right) f(z)\right]^{2}} \\
& +\gamma\left(1+\alpha_{1}\right) \frac{z H_{l, s}\left(\alpha_{1}+2\right) f(z)}{\left[H_{l, s}\left(\alpha_{1}\right) f(z)\right]^{2}}-2 \gamma \alpha_{1} \frac{z\left[H_{l, s}\left(\alpha_{1}+1\right) f(z)\right]^{2}}{\left[H_{l, s}\left(\alpha_{1}\right) f(z)\right]^{3}}
\end{aligned}
$$

is univalent in $U$, and

$$
\begin{gathered}
q_{1}(z)+\gamma z q_{1}^{\prime}(z) \prec\left[1+\gamma\left(\alpha_{1}-1\right)\right] \frac{z H_{l, s}\left(\alpha_{1}+1\right) f(z)}{\left[H_{l, s}\left(\alpha_{1}\right) f(z)\right]^{2}}+ \\
\gamma\left(1+\alpha_{1}\right) \frac{z H_{l, s}\left(\alpha_{1}+2\right) f(z)}{\left[H_{l, s}\left(\alpha_{1}\right) f(z)\right]^{2}}-2 \gamma \alpha_{1} \frac{z\left[H_{l, s}\left(\alpha_{1}+1\right) f(z)\right]^{2}}{\left[H_{l, s}\left(\alpha_{1}\right) f(z)\right]^{3}} \prec q_{2}(z)+\gamma z q_{2}^{\prime}(z),
\end{gathered}
$$

then

$$
q_{1}(z) \prec \frac{z H_{l, s}\left(\alpha_{1}+1\right) f(z)}{\left[H_{l, s}\left(\alpha_{1}\right) f(z)\right]^{2}} \prec q_{2}(z)
$$

and $q_{1}$ and $q_{2}$ are respectively the best subordinant and the best dominant.

Combining Corollary 11 and Corollary 15, we get the following sandwich result which improves the result obtained by Shanmugam et al. [16, Corollary 4.6].

Corollary 16. Let $\gamma \in C$ with $\operatorname{Re} \gamma>0, q_{1}$ be convex univalent in $U$ and $q_{2}$ be univalent in $U, q_{2}(0)=1$ and satisfies (3.1). If $f \in A, \frac{z L(a+1, c) f(z)}{[L(a, c) f(z)]^{2}} \in$ $H[1,1] \cap Q$,

$$
\begin{aligned}
{[1+\gamma(a-1)] } & \frac{z L(a+1, c) f(z)}{[L(a, c) f(z)]^{2}} \\
& +\gamma(1+a) \frac{z L(a+2, c) f(z)}{[L(a, c) f(z)]^{2}}-2 \gamma a \frac{z[L(a+1, c) f(z)]^{2}}{[L(a, c) f(z)]^{3}}
\end{aligned}
$$


is univalent in $U$, and

$$
\begin{aligned}
q_{1}(z)+\gamma z q_{1}^{\prime}(z) & \prec\left[1+\gamma\left(\alpha_{1}-1\right)\right] \frac{z L(a+1, c) f(z)}{[L(a, c) f(z)]^{2}}+\gamma(1+a) \frac{z L(a+2, c) f(z)}{[L(a, c) f(z)]^{2}} \\
& -2 \gamma a \frac{z[L(a+1, c) f(z)]^{2}}{[L(a, c) f(z)]^{3}} \prec q_{2}(z)+\gamma z q_{2}^{\prime}(z),
\end{aligned}
$$

then

$$
q_{1}(z) \prec \frac{z L(a+1, c) f(z)}{[L(a, c) f(z)]^{2}} \prec q_{2}(z),
$$

and $q_{1}$ and $q_{2}$ are respectively the best subordinant and the best dominant.

Acknowledgement. The authors would like to thank the referee of the paper for his helpful suggestions.

\section{REFERENCES}

[1] Ali, R. M., Ravichandran, V. and Subramanian, K. G., Differential sandwich theorems for certain analytic functions, Far East J. Math. Sci. 15 (2004), no. 1, 87-94.

[2] Bernardi, S. D., Convex and starlike univalent functions, Trans. Amer. Math. Soc. 135 (1969), 429-446.

[3] Bulboacă, T., Classes of first order differential superordinations, Demonstratio Math. 35 (2002), no. 2, 287-292.

[4] Bulboacă, T., Differential Subordinations and Superordinations, Recent Results, House of Scientific Book Publ., Cluj-Napoca, 2005.

[5] Bulboacă, T., A class of superordination-preserving integral operators, Indag. Math. (N. S.) 13 (2002), no. 3, 301-311.

[6] Carlson, B. C. and Shaffer, D. B., Starlike and prestarlike hypergeometric functions, SIAM J. Math. Anal. 15 (1984), 737-745.

[7] Cho, N. E., Kwon, O. H. and Srivastava, H. M., Inclusion and argument properties for certain subclasses of multivalent functions associated with a family of linear operators, J. Math. Anal. Appl. 292 (2004), 470-483.

[8] Choi, J. H., Saigo, M. and Srivastava, H. M., Some inclusion properties of a certain family of integral operators, J. Math. Anal. Appl. 276 (2002), 432-445.

[9] Dziok, J., Srivastava, H. M., Classes of analytic functions associated with the generalized hypergeometric function, Appl. Math. Comput. 103 (1999), 1-13.

[10] Dziok, J., Srivastava, H. M., Certain subclasses of analytic functions associated with the generalized hypergeometric function, Integral Transform. Spec. Funct. 14 (2003), $7-18$.

[11] Libera, R. J., Some classes of regular univalent functions, Proc. Amer. Math. Soc. 16 (1965), 755-658.

[12] Miller, S. S., Mocanu, P. T., Differential subordinations and univalent functions, Michigan Math. J. 28 (1981), no. 2, 157-171.

[13] Miller, S. S., Mocanu, P. T., Differential Subordination. Theory and Applications, Marcel Dekker Inc., New York, 2000.

[14] Miller, S. S., Mocanu, P. T., Subordinates of differential superordinations, Complex Variables 48 (2003), no. 10, 815-826.

[15] Ruscheweyh, St., New criteria for univalent functions, Proc. Amer. Math. Soc. 49 (1975), 109-115. 
[16] Shanmugam, T. N., Ravichandran, V. and Sivasubramanian, S., Differential sandwich theorems for some subclasses of analytic functions, Aust. J. Math. Anal. Appl. 3 (2006), no. 1, Art. 8, 11 pp. (electronic).

[17] Srivastava, H. M., Karlsson, P. W., Multiple Gaussian Hypergeometric Series, Ellis Horwood Ltd., Chichester, Halsted Press (John Wiley \& Sons, Inc.), New York, 1985.

[18] Tuneski, N., On certain sufficient conditions for starlikeness, Int. J. Math. Math. Sci. 23 (2000), no. 8, 521-527.

M. K. Aouf

Department of Mathematics

Faculty of Science

Mansoura University

Mansoura 35516, Egypt

e-mail: mkaouf127@yahoo.com

R. El-Ashwah

Department of Mathematics

Faculty of Science

Mansoura University

Mansoura 35516, Egypt

e-mail: r_elashwah@yahoo.com

Received July 22, 2008

\author{
A. O. Mostafa \\ Department of Mathematics \\ Faculty of Science \\ Mansoura University \\ Mansoura 35516, Egypt \\ e-mail: adelaeg254@yahoo.com
}

